\title{
AUTOMATIC SCHEME SELECTION FOR TOOLKIT HEX MESHIN
}

David R. White

Sandia National Laboratories, Albuquerque, NM. drwhite@sandia.gov

Timothy J. Tautges

Sandia National Laboratories, Albuquerque, NM. tjtautg@sandia.gov'

\begin{abstract}
Current hexahedral mesh generation techniques rely on a set of meshing tools, which when combined with geometry decomposition leads to an adequate mesh generation process. Of these tools, sweeping tends to be the workhorse algorithm, accounting for at least $50 \%$ of most meshing applications. Constraints which must be met for a volume to be sweepable are derived, and it is proven that these constraints are necessary but not sufficient conditions for sweepability. This paper also describes a new algorithm for detecting extruded or sweepable geometries. This algorithm, based on these constraints, uses topological and local geometric information, and is more robust than feature recognition-based algorithms. A method for computing sweep dependencies in volume assemblies is also given. The auto sweep detect and sweep grouping algorithms have been used to reduce interactive user time required to generate all-hexahedral meshes by filtering out non-sweepable volumes needing further decomposition and by allowing concurrent meshing of independent sweep groups. Parts of the auto sweep detect algorithm have also been used to identify independent sweep paths, for use in volume-based interval assignment.
\end{abstract}

Keywords: Cubit, mapping, submapping, sweeping, hexahedra, toolkit

${ }^{1}$ SANDIA IS A MULTIPROGRAM LABORATORY OPERATED BY SANDIA CORPORATION, A LOCKHEED MARTIN COMPANY, FOR THE UNITED STATES DEPARTMENT OF ENERGY UNDER CONTRACT DE-AC04-94AL85000. 


\section{DISCLAIMER}

This report was prepared as an account of work sponsored by an agency of the United States Government. Neither the United States Government nor any agency thereof, nor any of their employees, make any warranty, express or implied, or assumes any legal liability or responsibility for the accuracy, completeness, or usefulness of any information, apparatus, product, or process disclosed, or represents that its use would not infringe privately owned rights. Reference herein to any specific commercial product, process, or service by trade name, trademark, manufacturer, or otherwise does not necessarily constitute or imply its endorsement, recommendation, or favoring by the United States Government or any agency thereof. The views and opinions of authors expressed herein do not necessarily state or reflect those of the United States Government or any agency thereof. 


\section{DISCLAIMER}

Portions of this document may be illegible in electronic image products. Images are produced from the best available original document. 


\section{INTRODUCTION}

The finite element method is used to simulate a wide variety of physical phenomena, for example heat transfer, structural mechanics, and computational fluid dynamics. In recent years, mesh generation has emerged as one of the major bottlenecks in the simulation process. Although a high degree of automation is available in tetrahedral mesh generators, hexahedral mesh generators still require a great deal of user intervention. Since it is widely believed that hexahedral meshes are more accurate and more robust for some types of finite element analysis, especially in the non-linear regime, these types of meshes are more commonly used.

There has been a great deal of research into automated, all-hexahedral meshing algorithms[1][2][3], but as yet no algorithm has been found with the key characteristics of high robustness, high mesh quality and low element count. Therefore, current hexahedral mesh generation techniques rely on a set of simpler tools, which when combined with geometry decomposition leads to an adequate mesh generation process. The meshing algorithms in these tools include mapping/submapping[4], primitive templates[5], and sweeping or extrusion[6]. Of these, sweeping tends to be the workhorse algorithm, usually accounting for at least $50 \%$ of most meshing applications.

The sweeping algorithm involves extruding a set of quadrilaterals into a third dimension, producing a hexahedral mesh. The cross-section of the geometry being meshed can vary along the sweep direction, and the number of quadrilaterals in the set being swept can vary as well. Figure 1 shows several sweepable geometries. Many of the commercial mesh generation software packages currently include some form of sweeping algorithm[7][8], and varieties of this algorithm are reported elsewhere in the literature[6][9][10].

While sweeping is a widely used algorithm, it is not very automated. Before a volume can be "swept", the algorithm must be provided with input about which surface meshes are being swept along which side surfaces, and for how far. In practice, the process of determining and specifying these source/target surfaces is user-intensive and error prone. In order to increase the level of automation in all-hexahedral meshing, an automatic method for determining sweep directions and source/target surfaces is needed.

The detection of swept features has been studied in the feature recognition community for some time[11][12]. However, the resulting algorithms are usually geometry-based, relying on arrangements such as parallel surfaces for detecting the features. These arrangements represent geometric constraints placed on extruded volumes that that are determined by the application, for example solids to be manufactured by machining.

This paper describes a new algorithm for detecting extruded or sweepable geometries. This algorithm is based on topological and local geometric criteria, and is more robust than feature recognition-based algorithms. This algorithm has been implemented in 
the CUBIT mesh generation toolkit[13], and has been shown to work for virtually all sweepable geometries. This algorithm could also be used to detect extruded features for the purposes of feature extraction and dissembly planning.

This paper is arranged as follows. Definitions used in describing this algorithm are given in section 2; the characteristics that make a geometry sweepable (also referred to as 2.5D) are discussed in section 3; the auto sweep detection algorithm is described in section 4, while an algorithm for grouping volumes with sweep dependencies is described in section 5; examples are given in section 6. Conclusions are given in section 7 .

\section{BACKGROUND AND DEFINITIONS}

We begin with a geometric solid defined by a BREP or boundary representation model, for example as described in Mortensen [14]. Before describing the automatic sweep detect algorithm, we must define several geometric and topological characteristics used in the algorithm. These characteristics either determine local (e.g. surface) meshability, or they combine local information across collections of surfaces to form meta-information about the volume being meshed. These characteristics will be used in the next section to define the auto sweep detect algorithm.

(Surface) Vertex Type: The classification of a vertex by the type of quadrilateral mesh in the vicinity of that vertex. Four vertex types are used: End, Side, Corner, and Reversal. The mesh topology represented by each vertex type is shown in Figure 2 . To each vertex type, an integer value is assigned; this integer is used to evaluate meshability (this will be described shortly). The integers assigned to the vertex types End, Side, Comer and Reversal are $+1,0,-1$, and -2 , respectively.

Structured Mesh: In 2D, an all-quadrilateral mesh, where interior nodes (i.e. nodes not on the bounding loop(s)) are shared by exactly four quadrilaterals. In 3D, a structured mesh is one whose interior nodes are shared by exactly eight hexahedra.

Mapped Mesh: In 2D, a structured mesh whose boundary consists of exactly four End-type vertices or nodes, each shared by a single quadrilateral in that mesh, and any remaining exterior vertices and/or nodes shared by exactly two quadrilaterals. In $3 \mathrm{D}$, a structured mesh which contains exactly eight End-type vertices or nodes, each shared by a single hexahedron in the mesh, and remaining exterior vertices and/or nodes shared by either two or four hexahedra, depending on whether they fall on the exterior or interior of the bounding $2 \mathrm{D}$ mapped meshes.

Discussion: 
In a 2D mapped mesh, the exterior nodes and edges between a pair of Ends make up a "side" of the map; since mapped meshes contain exactly four Ends, they also contain exactly four sides. Ends typically fall on geometric vertices, although this is not required ${ }^{2}$. Sides are made up of one or more geometric edges, since in a BREP model edges bound faces.

Submapped Mesh: In 2D, a structured mesh whose exterior vertices and nodes include Comer- and Reversal-type as well as End-type vertices and/or nodes.

\section{Discussion:}

The submapping algorithm produces structured meshes[4]; it differs from the standard "mapping" or TFI algorithm in that it admits surfaces with more than four sides. It has been shown in [4] that a surface can be meshed with the submapping algorithm if all its vertices can be classified with a distinct vertex type, and the sum of vertex types is four. Thus, to determine whether a surface can be submapped, the vertex types must be computed and summed. Note that this is a geometric operation that is local to the surface.

In many cases, the angle used to determine vertex type is not an integer multiple of 90 degrees. Therefore, a "fuzzy" range around those integer multiples is used. If the angle is within a tolerance value (epsilon) of $90 \mathrm{n}$ degrees, the vertex is assigned the appropriate vertex type. The more this angle deviates from 90n degrees, the more distortion will be present at the quadrilateral(s) sharing that vertex or node. Note that the vertex type uniquely determines the topology of the mesh sharing that vertex, and therefore can be used to determine the topology of the overall mesh.

Link: A datastructure used to store sets of edges of a submapped surface according to their parameter directions.

\section{Discussion:}

A submappable surface can be assigned a local parameter space, with two parametric directions, referred to as $i$ and $j$; each edge on the surface can be assigned to one of these directions. For each parameter, there is a set of edges in each of the positive and negative parametric directions (e.g. $+i$ and $-i$ ); the classification of edges in these sets can be inferred from the choice of a reference vertex and the vertex types traversing around the surface. This is depicted in Figure 3.

Chain: A set of mappable and/or submappable surfaces which forms a complete loop in one of the parametric directions on the included surfaces.

Discussion:

\footnotetext{
${ }^{2}$ In the absence of a mesh, the vertex type is assigned using the angle of the edges meeting at that vertex; once a mesh exists, the mesh topology overrides any angle-based classification. This allows the placement of corner vertices in places not normally assigned End-type, e.g. on the boundary of a circular surface.
} 
A submappable surface can be traversed, either in the $i$ or $j$ direction, for example by going from the $-i$ edges to the $+i$ edges. Each $+i$ edge is shared by another surface in the volume; if that other surface is also submappable, it too can be traversed in the same way. If this process is continued, loops of submappable surfaces may emerge. We refer to a loop as a chain if and only if every edge in the parameter direction being traversed $(i$ or $j)$ is bounded by two surfaces, both of which are submappable and are part of the loop.

In the simplest case, a chain can be a single periodic surface (see Figure 4, Chain A) or a sequence of 4-sided mappable surfaces. However, because the surfaces are submappable and not simply mappable, a chain can also contain branches (see Figure 4, Chain B).

(Volume) Edge Type: analogous to surface vertex types, a classification of the mesh topology along that edge in a particular volume; uses the type classifications listed previously.

\section{Discussion:}

Like Surface Vertex Types, Volume Edge Types correspond directly to mesh topology in the region of the edge. The mesh represented by each edge type can be visualized by taking the meshes in Figure 2 and sweeping them into the third dimension; the geometric vertex gets swept into a geometric edge, which is assigned the same type as the vertex (End, Side, etc.). Thus, the geometric edge owns mesh edges, and each of these mesh edges is shared by two surface quads.

2.5D Volume Mesh: a volume mesh constructed by extruding one or more surface meshes into a third dimension.

\section{Discussion:}

A sweepable or $2.5 \mathrm{D}$ volume mesh contains one or more layers of hexahedra, with identical quadrilateral topology at each layer of the extrusion. One exception to this definition is if multiple source and/or target surfaces exist in the sweep; in these cases, groups of quadrilaterals are added to or removed from the sweep [10].

\section{WHAT MAKES A VOLUME 2.5D?}

We seek a precise definition of the geometric and topological conditions under which a solid can be meshed using extrusion (hereafter referred to as sweeping). These conditions can be deduced by observing the extrusion process.

In the following discussion, we will start by describing the topology of a swept mesh, but eventually will describe swept mesh constraints in terms of the geometric surfaces and edges of a volume and the mesh schemes and intervals assigned to those surfaces and edges. This allows us to evaluate the constraints before generating mesh for any of the bounding curves or surfaces. 
Where the terms edge and surface are used without qualification or clear context, they refer to the geometric entities rather than mesh.

By definition, extruding a logically one-dimensional segmented line into a second dimension results in another line with like topology; the line being swept is referred to as the source, while the other line is called the target. The extrusion can be done over several discreet steps or layers. with straight-line segments joining the vertices between adjacent layers. This is depicted in Figure 5, left. In three dimensions, a group of contiguous, logically two-dimensional mesh facets is swept into the third dimension, with each layer having identical mesh topology to the last and edges connecting corresponding vertices between layers. By the definition of sweeping a facetted line, as the two-dimensional facets are swept into the third dimension, the logically one-dimensional line(s) bounding them sweeps out one or more-structured surface meshes (see Figure 5, right). We summarize these statements in the following Lemma:

Lemma 1: Sweeping a contiguous set of mesh faces generates a) layers of mesh faces and mesh elements with identical topology, and b) side (linking) surface meshes that are structured.

Furthermore, the following results directly from this Lemma:

Corrolary: A swept mesh is bounded by one or more non-intersecting chains.

If the set of mesh faces being swept is divided into several regions of contiguous faces, and each region is swept a different number of layers, the resulting sweep is a multiple source/target sweep. In this case, Lemma 1 is modified to:

Lemma 1': Sweeping one or more contiguous sets of mesh faces into a third dimension generates:

a) layers of faces and elements which have identical topology except where contiguous regions of faces and corresponding elements have been completely added or removed from the sweep, and b) side (linking) surfaces which are mapped if the outer boundary of the regions being added or subtracted from the sweep do not intersect the original boundary of the sweep, or submapped if the boundaries do intersect.

Lemma l'a is obvious by inspection (e.g. generate a swept mesh as in Lemma la then remove one or more hexes, but not all hexes, from the first layer). Lemma I'b can be shown as follows. Consider the two cases; if the boundary of the set of faces being added or removed does not intersect the original boundary, then we are still sweeping that original boundary after the addition or removal, and the result is still a mapped mesh. On the other hand, if the two boundaries do intersect, then we are modifying the boundary of the original sweep; the parameter extents on the linking surface are also modified during the sweep, and by definition this generates a submapped mesh.

Using the definitions of volume edge types, and Lemma 1'a and 1'b, we can also observe that: 
Lemma 2: In a swept volume:

a) edges between sourceitarget surfaces and linking surfaces are always of type End or Corner;

b) edges between source/target surfaces are always of type Side or Reversal;

c) edges between linking surfaces that are purpendicular to the sweep direction (i.e. edges that bound a single layer) are always of type Side or Reversal; and

d) edges between linking surfaces that are parallel to the sweep direction (i.e. edges that bound multiple layers) can be any type.

Next, we state the following:

Lemma 3: Each set of contiguous source/target surfaces has a single, common ij parameter with respect to the bounding linking surface(s).

Lemma 3 follows from Lemma l'a and l'b and the definitions of mapped and submapped surfaces (and their parameterizations).

Lemma 4: Traversing from a set of contiguous source/target surfaces over any boundary edge of type End results in traversing a linking surface in the same ij parameter direction (i.e. $i$ or $j$ ), no matter which End-type edge is traversed; similarly for traversing any Comer-type edge.

This Lemma follows from Lemmas 2, 3 and 1'b.

Lemma 5: All linking surfaces in a swept mesh can be assigned a global, consistent ij parameterization.

Proof: By consistent, we mean that anywhere linking surfaces meet at a shared edge, the type ( $i$ or $j$ ) and value of ij parameter along that edge is the same. For linking surfaces which are part of the same chain, a consistent parameterization exists because the surfaces are mapped or submapped and, by the definition of a chain, the surfaces in the chain can be unrolled to form a contiguous submapped or mapped mesh. The parameterization of one chain extends to chains whose surfaces share edges with surfaces in that chain. Disjoint chains have related parameterizations by virtue of Lemma 3.

Lemma 6: Traversing from the same set of contiguous source/target surface(s) onto a linking surface over and End-type versus a Corner-type edge will result in traversing the same ij parameter in opposite directions (e.g. $+i$ and $-i$ ) on the linking surface.

Proof: Existence of a Corner-type edge implies the addition or removal of a region of faces from the source/target surface(s) being swept (by Lemma 1'a and the definition of a Corner-type edge). Consider the geometry in Figure 6 (upper), with source surface $S_{1}$, linking surface $L_{1}$, and bounding edges $C_{1}$ and $E_{1}$ of type Comer and End, respectively. If $L_{1}$ is extended into the volume, the volume is separated into two pieces which share a surface. Traversing from $S_{1}$ to $L_{1}$ over $C_{1}$ is indicated by arrow a in Figure 6 (lower), while traversing from $S_{1}$ to $L_{1}$ over $E_{1}$ is indicated by arrow $b$. However, by Lemma 4 , this is equivalent to 
traversing along arrow $\mathfrak{c}$; because the interface is shared by both volumes, this in turn is equivalent to traversing along $d$. From here, it is clear that arrows $\mathbf{a}$ and $\mathbf{d}$ point in opposite directions along the same ij parameter of the linking surface. Using Lemmas 3 and 5, we can prove this is true even in the case where the boundary loops containing the Comer-type and End-type edges do not intersect.

Lemmas 1.6 state several characteristics of a swept mesh; to be sweepable, a volume must at least show these characteristics. We summarize this conclusion in the following theorem:

Theorem 1: A volume is sweepable only if:

a) the volume has one or more non-intersecting chains;

b) for each set of contiguous sourceftarget surfaces, traversing over all E-type or C-type edges bounding the set results in traversing in the same global ij parameter and direction on the linking surface(s);

c) for each set of contiguous source/target surfaces bounded by both E-type and C-type edges, traversing over any E-type edge bounding the set results in traversing in the same global ij parameter and opposite direction on the linking surface as resulting from traversing over any $C$-type edge bounding the set.

Proof: a) was proven by Lemma 1; b) and c) were proven by Lemma 6.

Although the conditions in Theorem 1 are necessary for sweepability, these conditions are not sufficient, even if we place no constraints on the quality of the resulting mesh. For example, consider a volume containing a through-hole, one which ties itself in a knot without its surfaces intersecting. This volume is clearly not sweepable, but still passes the constraints in Theorem 1 . There is likely an additional constraint that can be placed on the bounding surface mesh which would capture examples like this; we conjecture that this constraint is similar to those discussed in [18]. However, the authors have never observed an example like this in practice, nor expect to see any like this in practical applications. 
Although we have proven that Theorem 1 is a necessary condition for sweepability, looking at an example will show why Theorem $l b$ and $1 \mathrm{c}$ in particular are needed. Consider the volume in Figure 7. If the edges $E_{1}$ and $E_{2}$ are designated as type End, the surfaces $L_{1}$ and $L_{2}$ each form a chain, these chains are non-intersecting, and by Theorem la the volume is sweepable. However, traversing from $S_{1}$ to $L_{1}$ and $L_{2}$ over $E_{1}$ and $E_{2}$, respectively, results in traversing the linking surfaces in opposite directions in the same ij parameter; this violates Theorem $\mathrm{lb}$. On the other hand, if edge $\mathrm{E}_{1}$ is designated type Corner, then according to Theorem $1 b$ and $l c$ the volume is sweepable. In fact, the volume can be meshed by sweeping $S_{2}$ down to to $E_{1}$, then adding surface $S_{1}$ and sweeping down to $S_{3}$.

When the type of edge E1 in Figure 7 is set to Comer, the volume becomes sweepable. However, because edge E2 is type end, quadrilateral pairs sharing edges on E2 will be part of the same hex; the dihedral angle between these faces will be 180 degrees. For most applications, hexes with interior angles of 180 degrees are of insufficient quality. Therefore, even though volumes meeting the conditions in Theorem 1 are sweepable, the resulting meshes may not be of sufficient quality for analysis. Additional constraints are needed to improve mesh quality.

The combination of volume edge types and source/target surfaces uniquely determine the topology of the mesh at all geometric edges. This topology was described in the definition of the different volume edge types, and constraints on allowable volume edge types were described in Lemma 2. For the purposes of mesh quality, we must place constraints on the dihedral angles between surfaces meeting at various edge types; these constraints are similar to those used in the submapping algorithm [4].

Assuming that an ideal hex element is one whose angles are all 90 degrees, each of the vertex types in Figure 2 (and the corresponding volume edge types) has a corresponding ideal angle; these angles are 90, 180, 270 and 360 degrees for End, Side, Comer and Reversal types, respectively. Around these ranges are "fuzzy" regions, approximately 45 degrees on either side of the ideal, within which hex quality is degraded but not inadequate. Most finite element applications require that face angles be bounded between zero and 180 degrees. These angle constraints impose limits on angles at the given edge types. The angle at End-type edges is limited to 180 degrees; Side-type edges are limited to 360 degrees. Corner and Reversal type edges are limited to 360 degrees, not to prevent hex angles greater than 180 degrees but to prevent overlapping elements. These element constraints are summarized in the following Theorem:

Theorem 2: To ensure element angles less than 180 degrees and non-overlapping elements, End-type edges must be less than 180 degrees, and Side, Comer and Reversal edges must be less than 360 degrees. All angles must be greater than zero degrees.

Finally, the available sweep algorithm may determine additional constraints on the topology of surfaces in $S$ or $L$. The "standard" sweep algorithm allows the extrusion of one surface (the "source" surface) onto a single "target" surface of identical topology; this is often referred to as a one-to-one sweep[9][15]. There also exist other varieties of sweeping which have fewer constraints on source and target topology, e.g. "many-to-one" or n->1 sweeps[16], and "many-to-many" or n->m sweeps[10][17]. 
Many-to-many sweeps may require modifications to the toplogy of source or target surfaces, depending on implementation[17]. We summarize these notes in the following Lemma:

Lemma 3: Volumes containing surfaces in $S$ sharing an edge of type Reversal require muliple target sweeping; volumes containing surfaces in $S$ and $L$ sharing edges of type Comer reguire multiple source/single target or multiple source/target sweeping; volumes containing surfaces in $L$ which share edges purpendicular to the sweep direction of type Reversal require multiple source/single target sweeping; and volumes containing multiple surfaces in $S$ require multiple source and possibly multiple target sweeping.

Some algorithms may also impose topological restrictions on linking surfaces; for example, some implementations require the linking surfaces to all be mappable (having only 4 sides), while others allow more general submappable surfaces with imprints which form interior holes. These additional constraints can be evaluated after the determination of whether a volume is sweepable. These considerations lead to a fourth type of sweepability criterion:

Lemma 4: Constraints on the topology of linking surfaces (implementation dependent).

Fuzzy regions around the ideal angles for the four vertex and edge types allow the use of submapping and mapping schemes on surfaces, and sweeping on volumes, where they might not typically be used. For example, the surface mesh shown in Figure 8 was obtained by setting vertex 4 to type End, even though by angle measurement it was closer to type Side. Although this degraded mesh quality locally, it allowed the volume to be swept, and the resulting mesh had an acceptable overall mesh quality.

For application to mesh generation, the definition of a sweepable volume is less constrained geometrically, since it is not strictly required that the cross section of the extrusion have a constant area or even that its normal maintain a constant direction. This makes the detection of mesh-sweepable volumes considerably more efficient, since there is no need to evaluate global geometric constraints. At the same time, an extruded volume in the mesh generation application is more constrained in terms of topology, as represented by Theorems 1 and 3 above. There are also local geometric constraints, represented by Theorem 2 . However, topological and local geometric constraints are easier and faster to evaluate than the global geometric constraints used in the past.

\section{AUTO SWEEP DETECTION ALGORITHM}

Theorem 1 in the previous section identified the conditions necessary for a volume to be sweepable. In this section, we describe the algorithm used to verify that the conditions in Theorem 1 are met by a given volume. The auto sweep detection algorithm consists of the following four steps:

1) Classify surface mesh schemes locally using surface vertex types. 
Automatic surface mesh scheme assignment is accomplished by first computing surface vertex types for all vertices on a surface, then summing the angles at the vertices (assigning the numbers $1,0,-1$ and -2 to end, side, comer, and reversal vertex types, respectively). If this sum is equal to four, the surface is submappable[4]. Note that the criteria used to assign vertex types for automatic surface mesh scheme assignment are somewhat more conservative than those used to find the "comers" in the submap algorithm when the user assigns that scheme explicitly. More conservative vertex type criteria lead to fewer surfaces that are assigned a submap scheme; the surfaces not assigned submap are usually assigned the paving scheme[19].

\section{2) Group mappable and submappable surfaces into chains.}

Once the comers have been chosen, mapped and submapped surfaces have an implied, logical 2D parameter space defined. This parameter space is implied even before meshing occurs, since the corners define the structure of the mesh if not the actual mesh. This parameterization can be used to traverse from a given edge across the surface to the "opposite" edge (see Figure 3). This traversal can continue across the surface sharing the second edge, and so on until either it meets up with the original edge again or it encounters a surface that is not assigned the map or submap mesh scheme. If a complete loop of surfaces results, this group of surfaces define a chain.

If a submapped surface is encountered during a traversal. the loop may branch into more than one section; this branching is analogous to a parallel circuit. For example, Figure 3 shows one such chain, part of which branches into two parallel sections (parallel in a topological sense, not a geometric sense).

The result of looping the surfaces is a number of chains. If the number of chains is zero, there are no complete loops of mappable/submappable surfaces, therefore the volume is not sweepable. If on the other hand all the bounding surfaces of a volume are mappable or submappable, then the volume itself is submappable. Finally, there are many cases where a submappable surface is part of two chains, but the volume is not submappable. In these cases, one or more source/target surfaces will be mappable or submappable.

\section{3) Compute volume edge types.}

The computation of volume edge types is not done until looping the surfaces bounding the volume has been done; the cost of this step is saved in the cases where the volume is either submappable or when no chains are found. Note that computing volume edge types is an operation local to the edge and the two surfaces connected to it.

4) Traverse surfaces, grouping them in $S$ and $L$.

In addition to determining whether a volume is sweepable, it must be determined which are the source and target surfaces for the sweep. This is done by traversing the surfaces; pseudo code for this traversal process is shown in 
Table 1.

There are several things to note in the pseudo code shown in Table 1. First, the traversal of source/target and linking surfaces is recursive, and ends when either all the surfaces have been put into one of the lists ( $\mathrm{S}, \mathrm{T}$ or $\mathrm{L})$, or a surface is encountered which should be a linking surface (i.e. submappable and part of a valid chain) but which is not.

The result of this traversal will be a determination of whether or not the volume is sweepable, and if so, what the source and target surfaces are. This latter information is useful for evaluating criteria in Theorems $1 \mathrm{~b}, 1 \mathrm{c}$ and $2-4$. The assignment of source and target lists is arbitrary; that is, these lists can be interchanged. This can be useful when the target surface(s) are meshed or when there are multiple targets and a single source (switching the lists in this case simplifies the sweep).

\section{AUTOMATIC SWEEP GROUPING}

After assigning volume sweep schemes, these volumes can be meshed individually. However, in many applications, the volumes are part of a larger assembly, where the volumes share surfaces and their corresponding mesh. In this case, there are additional constraints on the order of meshing volumes that must be met to avoid further complications in sweeping the volumes.

For example, consider the four volumes in Figure 9. If these volumes are meshed in an arbitrary order, for example volumes 1,2, 4 then 3 , the last volume will already have mesh on all its source and target surfaces specified. Changing the mesh on these surfaces is not possible without changing the adjoining volume meshes, which can be difficult. Therefore, meshing volume 3 will be difficult because the source and target meshes will have to coincide. By Lemma 1'a, the number of faces on the source and target surfaces must be the same; if they are not, the volume will not be sweepable.

It is clear that the volumes in Figure 9 can be meshed in an order which avoids any of the above difficulties; for this example, the order is volume $3,4,2$ then 1 . In fact, it is not difficult to build this order automatically by traversing the connected volumes over their source and target surface lists. The algorithm for this traversal, referred to as "automatic sweep grouping", is shown in 
Table 2.

Automatic sweep grouping is useful primarily for resolving dependencies in sweep order when sweeping assemblies of volumes. However, it is also useful for separating a large collection of volumes into independent groups, each of which can be meshed independently of the others ${ }^{3}$. This facilitates the meshing of large assemblies by teams of users, and has significantly reduced the wall clock time for hex meshing these assemblies.

\section{DISCUSSION AND EXAMPLES}

There are many uses for an automatic sweep detection algorithm. First and foremost, the algorithm is useful for automating the specification of source and target surfaces for volumes that the user knows are sweepable. For example, the geometry shown in Figure 10 consists of 33 sweepable volumes; auto sweep detect determines that these volumes are sweepable, and sets the source and target surfaces for each automatically. Note that in this example, the block contains both blind holes and through-holes. More examples of sweepable volumes detected by this algorithm are shown in Figure 11, Figure 12 and Figure 13.

A less obvious application of auto sweep detect is to filter a large group of volumes into sweepable and non-sweepable ones; this allows the user to quickly determine which volumes are not meshable and need to be decomposed further (in the case where a 3D auto hex algorithm is not available). For example, on a large meshing application at Sandia, this algorithm identified 220 of 245 volumes as being sweepable, leaving only $10 \%$ of the volumes for further inspection and decomposition[20]. Thus, this algorithm can be used to quickly reduce the number of volumes needing further interactive decomposition.

The identification of chains of surfaces emerging in the sweeping process has also been used to identify independent sweep paths, which in turn is used to determine mesh interval constraints which guarantee that the resulting volume can be meshed[21]. This is useful when sweeping volumes containing through-holes, for example.

This algorithm is also useful for guiding an automatic geometry decomposition tool that is being developed[22]. Auto sweep detect can provide a stopping criterion for the decomposition tool, terminating decomposition when all the pieces are sweepable. Chains of surfaces bounding volumes which are not sweepable may also be useful for guiding further decomposition.

\footnotetext{
${ }^{3}$ These sweep groups are not completely independent, because they must satisfy interval constraints as described in [21]. However, these constraints can be satisfied before meshing begins; the mesh on linking and other interface surfaces is then deterministic, so it does not matter which volume owning one of these surfaces generates its mesh.
} 


\section{CONCLUSIONS}

In this paper we have listed conditions under which a volume is sweepable, and have proven that these conditions are necessary for sweepability. These conditions are listed in three Theorems, and are based on global topology and local geometry. We have also shown that these conditions are not sufficient for some cases, namely volumes whose genus is not zero.

An algorithm for determining whether or not a volume is sweepable, and if so, identifying the source and target surfaces, has also been described. This algorithm relies on local geometry in the form of vertex and edge types, as well as on surface topology. Because only local geometric information is used, the algorithm is quite efficient and reliable.

Finally, it has been shown that there are also sweep order dependencies which must be met to avoid difficulty when meshing assemblies of volumes; in some cases, not satisfying these dependencies can result in some of the volumes not being sweepable. An algorithm which computes these dependencies has also been given; this algorithm relies only on topology information for swept volumes, and is therefore quite efficient.

The auto sweep detect algorithm has been used to reduce interactive user time required to generate all-hexahedral meshes by filtering out non-sweepable volumes needing further decomposition. Parts of the algorithm have also been used to identify independent sweep paths, for use in volume-based interval assignment. The automatic sweep grouping algorithm has also been useful for resolving sweep dependencies, and therefore reducing interactive time meshing large assemblies. It has also been useful for splitting large meshing jobs into independent pieces which can be meshed concurrently by multiple users.

The auto sweep detect algorithm may also prove useful for guiding automatic geometry decomposition, both by providing a stopping criterion for such a tool, and for guiding further decomposition using completed chains on unsweepable volumes.

\section{REFERENCES}

[1] N. T. Folwell and S. A. Mitchell, Reliable Whisker Weaving via Curve Contraction', Proc. 7th Int. Meshing Roundtable, Sandia National Laboratories, Albuquerque, New Mexico, October 1998.

[2] A. Scheffer, M. Etzion, A. Rappoport and M. Bercovier, Hexahedral Mesh Generation Using the Embedded Voronoi Skeletons', Proc. 7th Int. Meshing Roundtable, Sandia National Laboratories, Albuquerque, New Mexico, October 1998.

[3] M. Muller-Hannemann, Hexahedral Mesh Generation by Successive Dual Cycle Elimination', Proc. 7th Int. Meshing Roundtable, Sandia National Laboratories, Albuquerque, New Mexico, October 1998.

[4] David R. White, 'Automated Hexahedral Mesh Generation by Virtual Decomposition, Proc. 5th Int. Meshing Roundtable, SAND96-2301, Sandia National Laboratories, Albuquerque, New Mexico, September 1996. 
[5] M. B. Stephenson and T. D. Blacker, 'Using Cojoint Meshing Primitives to Generate Quadrilateral and Herxahedral Elements in Irregular Regions', Proc. ASME Computations in Engineering Conference, 1989.

[6] Patrick M. Knupp, 'Applications of Mesh Smoothing: Copy, Morph, and Sweep on Unstructured Quadrilateral Meshes', Int. J. Numer. Meth. Eng., 45, 37-45 (1999).

[7] FEMAP, Enterprise Software Products, Inc., http://www.femap.com.

[8] ANSYS, ANSYS, Inc., http://www.ansys.com.

[9] M. L. Staten, S. A. Canann and S. J. Owen, 'BMsweep: Locating Interior Nodes During Sweeping', Proc. 7th Int. Meshing Roundtable, Sandia National Laboratories, Albuquerque, New Mexico, October 1998.

[10] T. Blacker, The Cooper Tool', Proc. 5th Int. Meshing Roundtable, SAND96-2301, Sandia National Laboratories, Albuquerque, New Mexico, September 1996.

[11] Somashekar Subrahmanyam and Michael Wozny, 'An Overview of Automatic Feature Recognition Techniques for Computer-Aided Process Planning', Computers in Industry 26 (1995) 1-21.

[12] Anshuman Razdan, Mark R. Henderson, Patrick F. Chavez and Paul A. Erickson, 'Feature Based Object Decomposition for Finite Element Meshing', The Visual Computer (1989) 5:291-303.

[13] T. D. Blacker et al., 'CUBIT mesh generation environment, Vol. 1: User's manual', SAND94-1100, Sandia National Laboratories, Albuquerque, New Mexico, May 1994.

[14] Michael E. Mortenson, 'Geometric Modeling', Wiley \& Sons, 1985.

[15] Amy P. Gilkey and Gregory D. Sjaardema, 'GEN3D: A GENESIS Database 2D to 3D Transformation Program', SAND890485, Sandia National Laboratories, Albuquerque, New Mexico, February 1994.

[16] Lai Mingwu, Steven E. Benzley, Greg Sjaardema and Tim Tautges, 'A Multiple Source and Target Sweeping Method for Generating All-Hexahedral Finite Element Meshes, Proc. 5th Int. Meshing Roundtable, SAND96-2301, Sandia National Laboratories, Albuquerque, New Mexico, September 1996.

[17] Lai Mingwu, Steven E. Benzley and David R. White, 'Automated Hexahedral Mesh Generation by Geneeralized Multiple Source to Multiple Target Sweeping, 2nd Symposium on Trends in Unstructured Mesh Generation, Boulder, Colorado, Aug. 4-6, 1999, to appear.

[18] Scott A. Mitchell, "A Characterization of the Quadrilateral Meshes of a Surface Which Admit a Compatible Hexahedral Mesh of the Enclosed Volume", Proceedings of the $13^{\text {th }}$ Annual Symposium on Theoretical Aspects of Computer Science, Springer, pp. 465-476, 1996.

[19] Roger J. Cass, Steven E. Benzley, Ray J. Meyers and Ted D. Blacker, “Generalized 3-D Paving: An Automated Quadrilateral Surface Mesh Generation Algorithm', Int. J. Numer. Meth. Eng., 39, 1475-1489 (1996). 
[20] P. Knupp, D. J. Melander, S. A. Mitchell, T. J. Tautges and D. R. White, Personal experience modeling the MC4380 Neutron Generator Tube, $7 / 98$.

[21] J. Shepherd, S. Benzley, S. A. Mitchell, Interval Assignment for Volumes with Holes, 2nd Symposium on Trends in Unstructured Mesh Generation, Boulder, Colorado, Aug. 4-6, 1999, to appear.

[22] Y. Lu, R. Gadh and T. J. Tautges, 'Feature Decomposition for Hexahedral Meshing', submitted to the 1999 ASME Design Automation Conference, Las Vegas, Nevada, Sept. 12-15, 1999. 


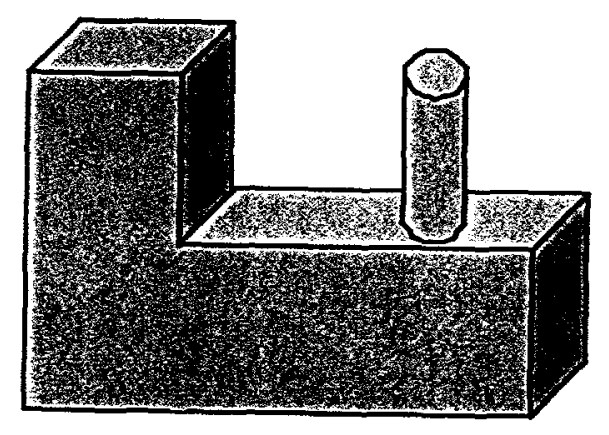

Figure 1: Sweepable geometries. 

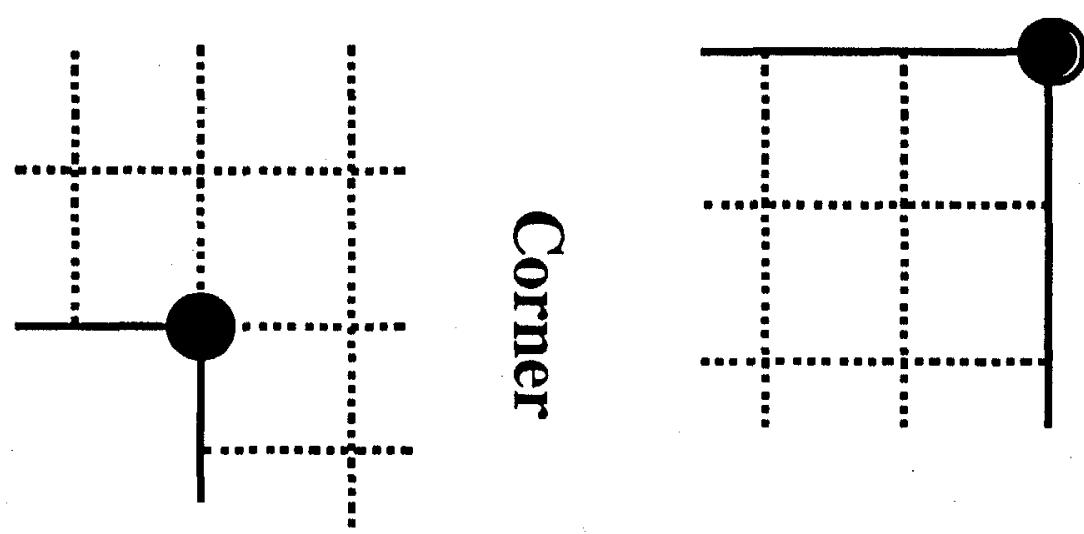

졸
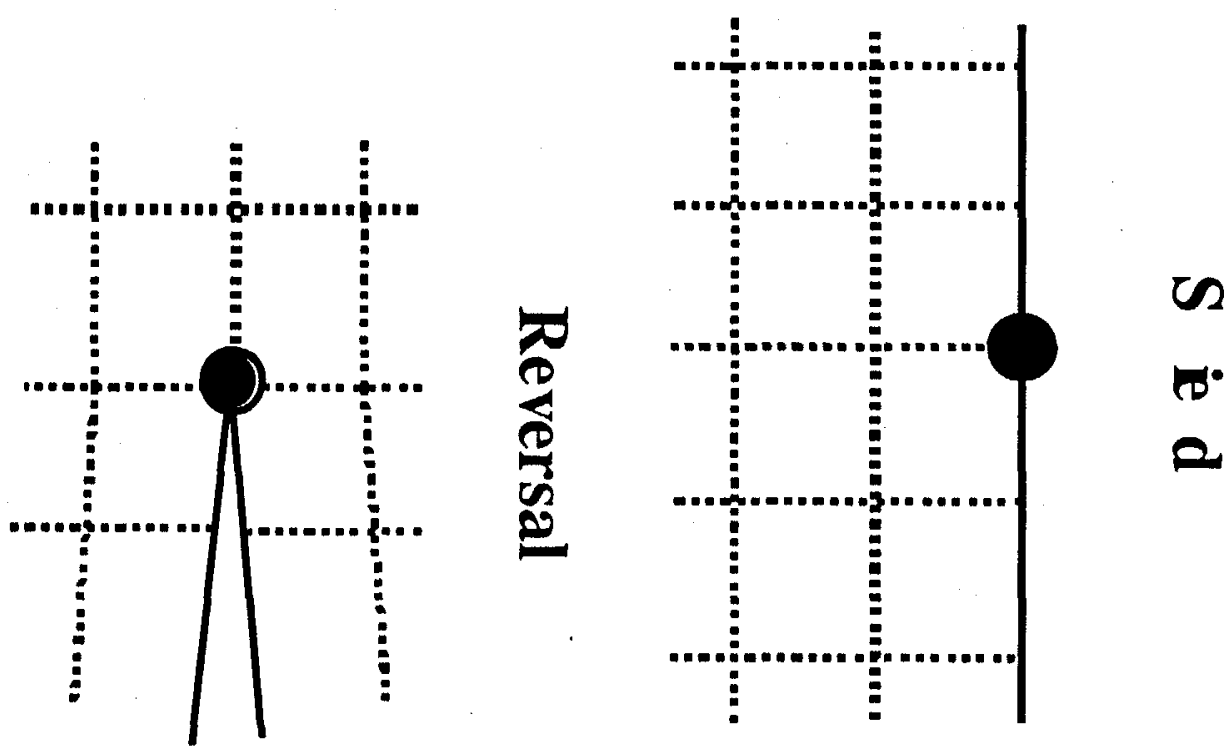


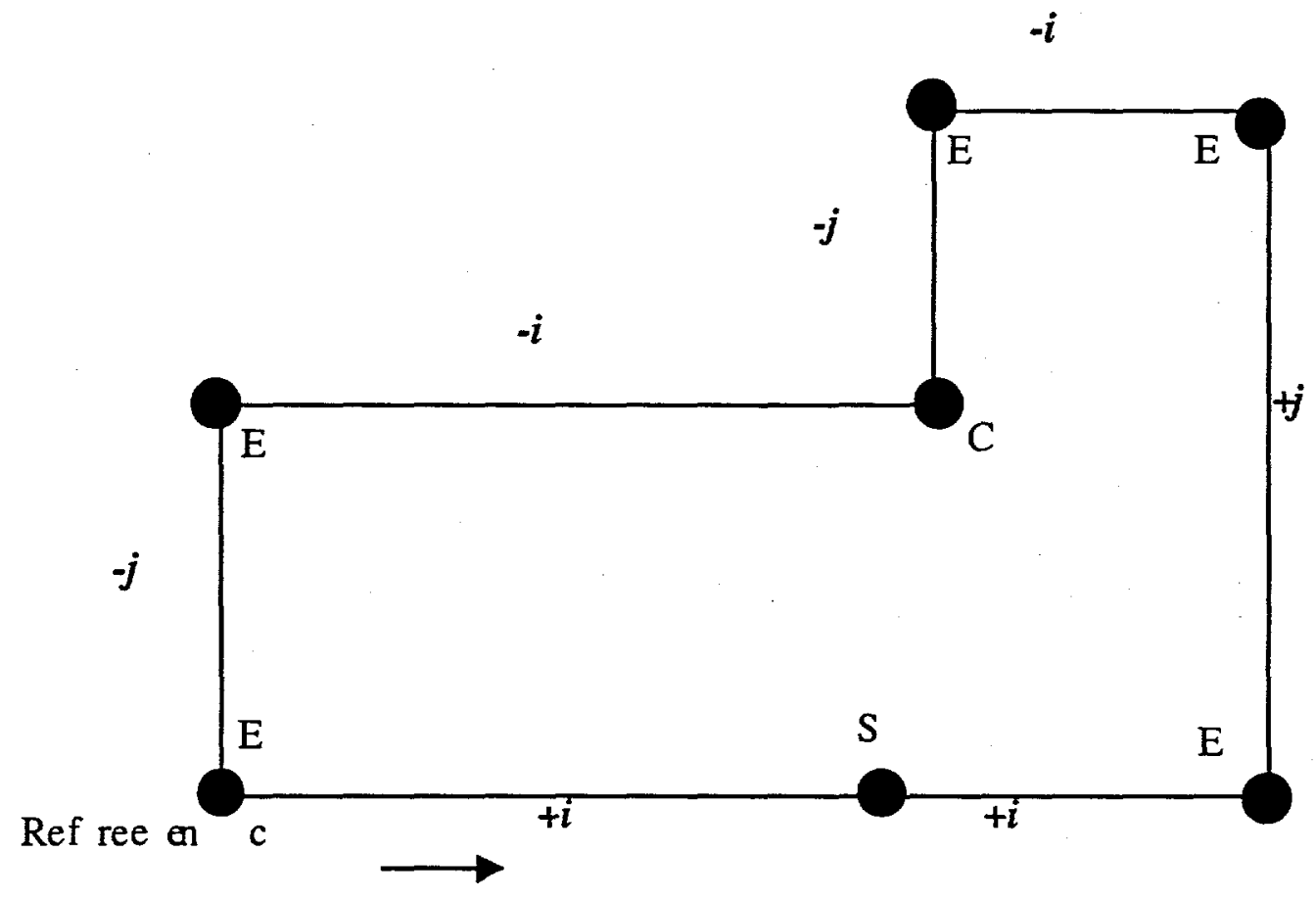

Figure 3: Submappable surface, showing vertex types ( $E=e n d, C=c o r n e r, ~ S=s i d e)$ and parametric directions $(+1-i,+1-j)$. 


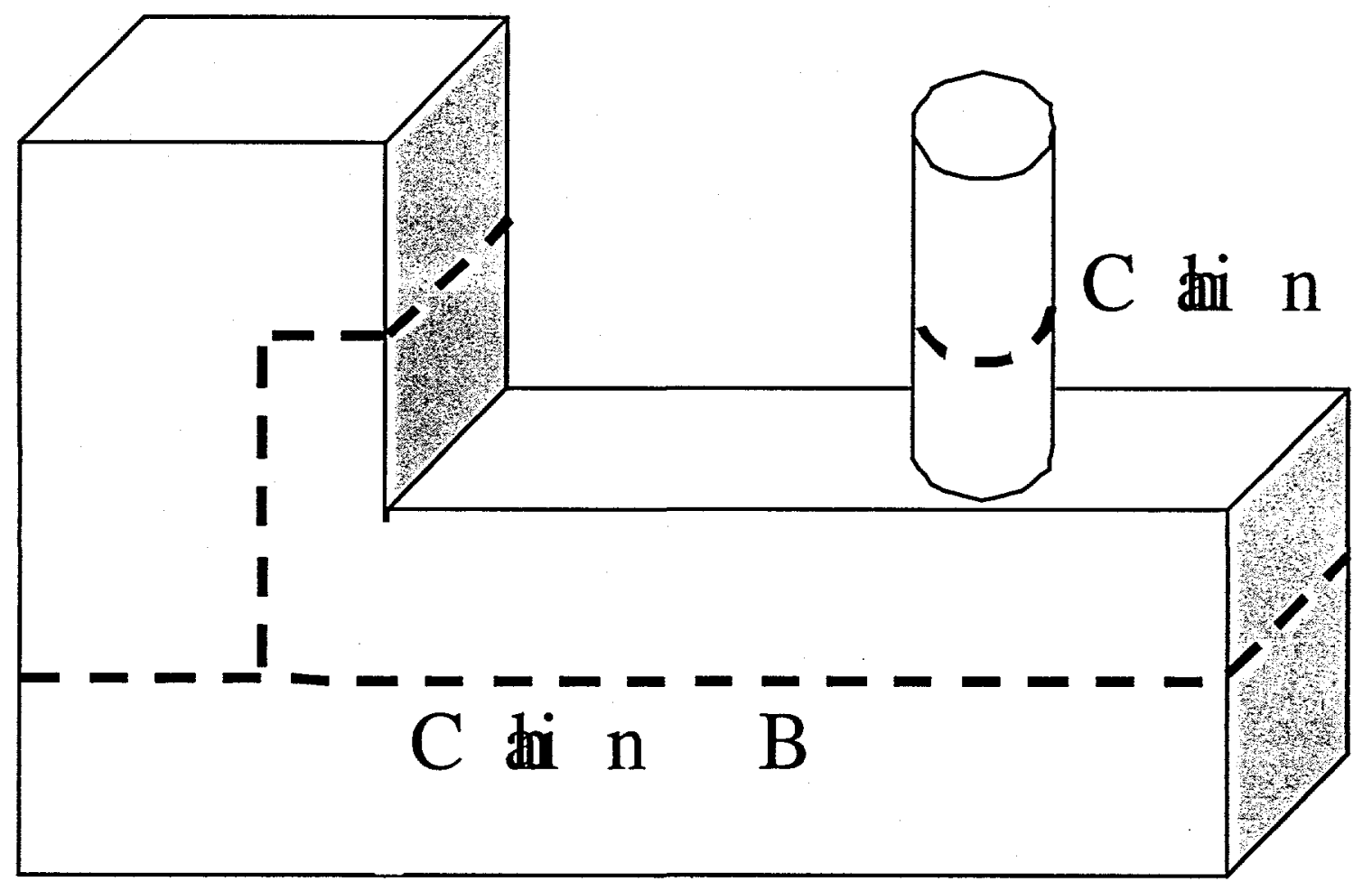

Figure 4: Chains on a solid; Chain $A$ is on a periodic surface ; Chain $B$ is branched. 

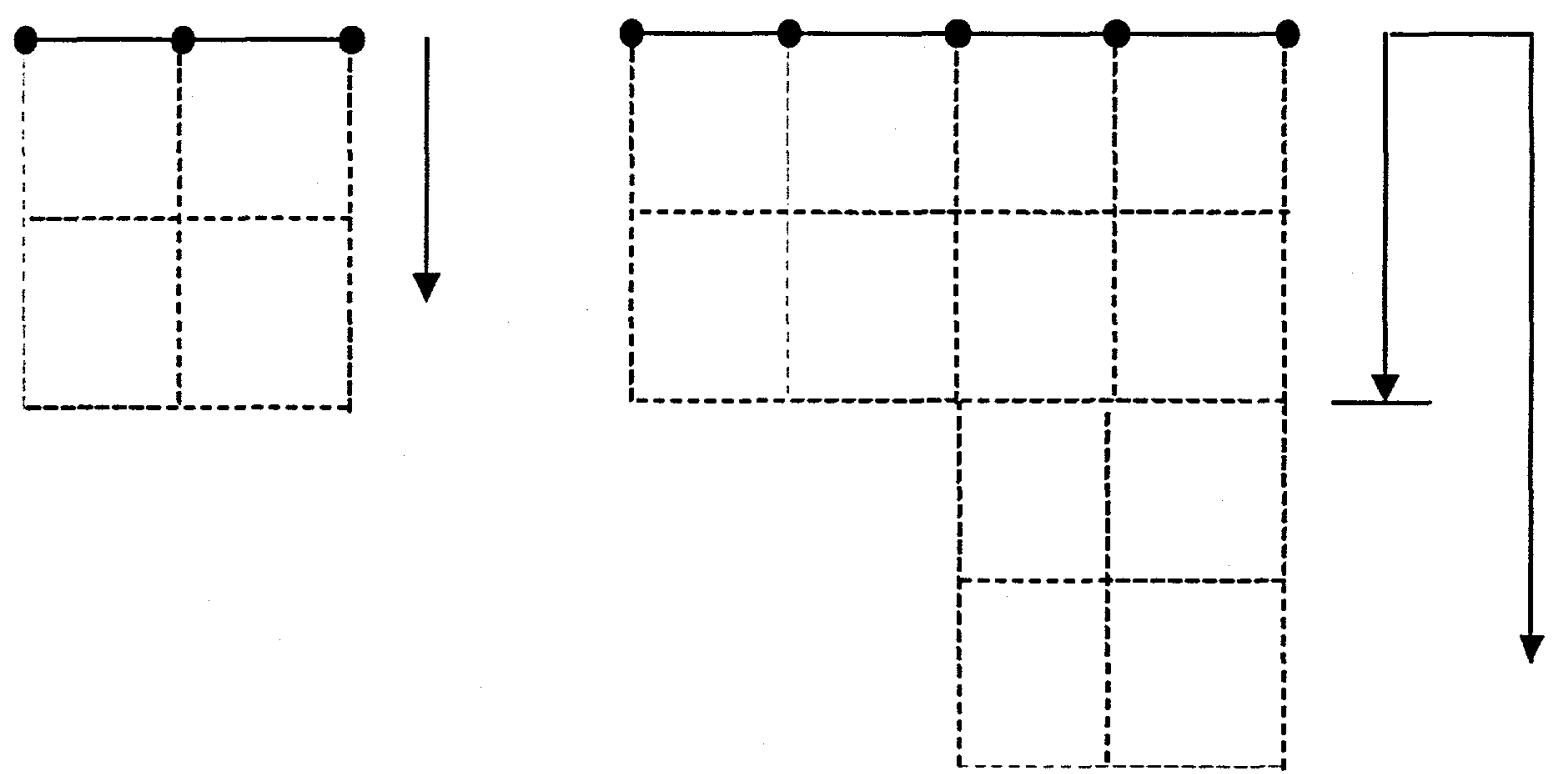

Figure 5: Extrusion of a facetted line into a mapped mesh (left); extrusion of different parts different distances into a submapped mesh (right). 

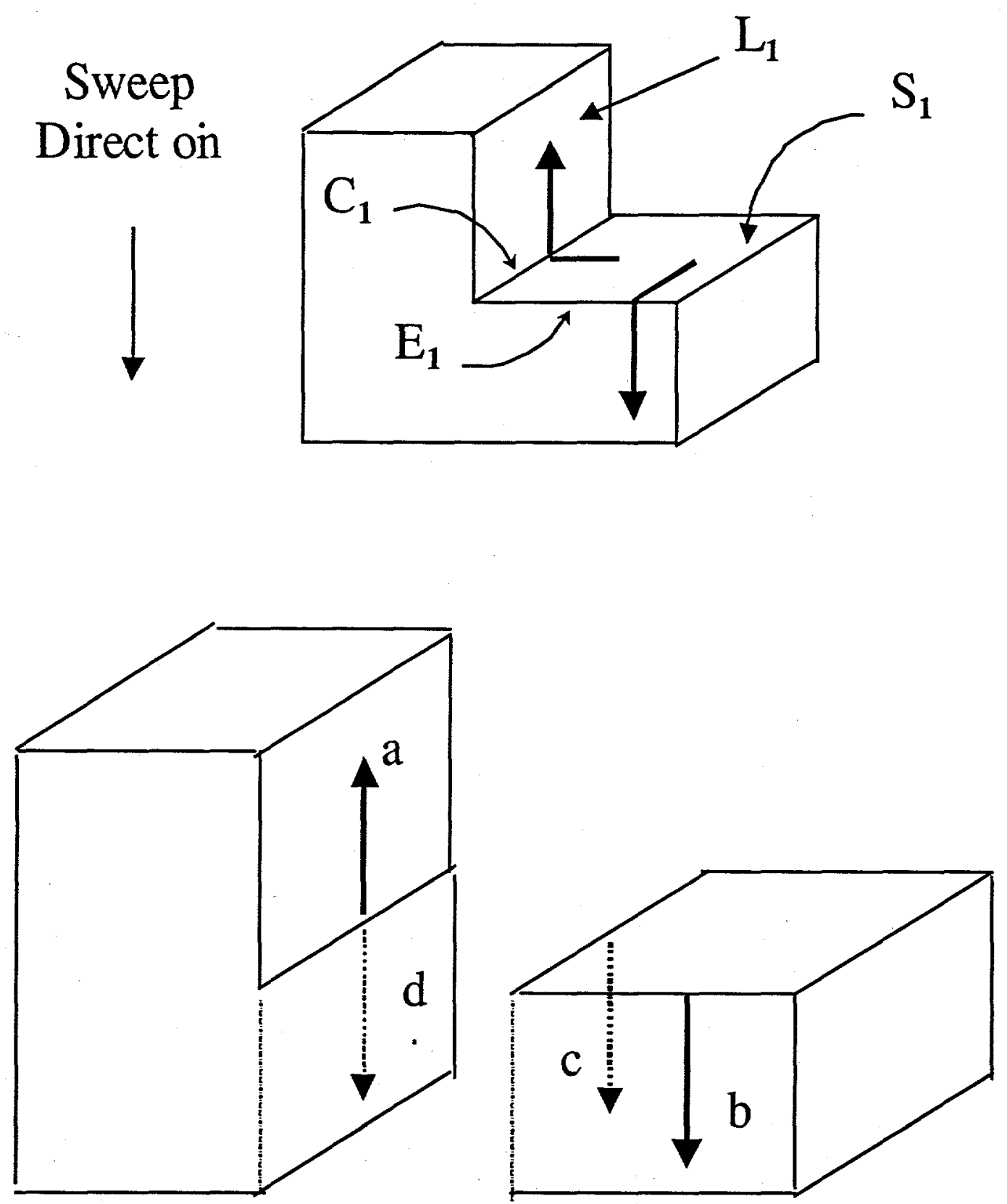

Figure 6: Traversing from a source surface over a Corner-type edge $\left(C_{1}\right)$ and End-type edge $\left(E_{1}\right)$ results in traversing the linking surface in opposite directions along the same ij parameter. 


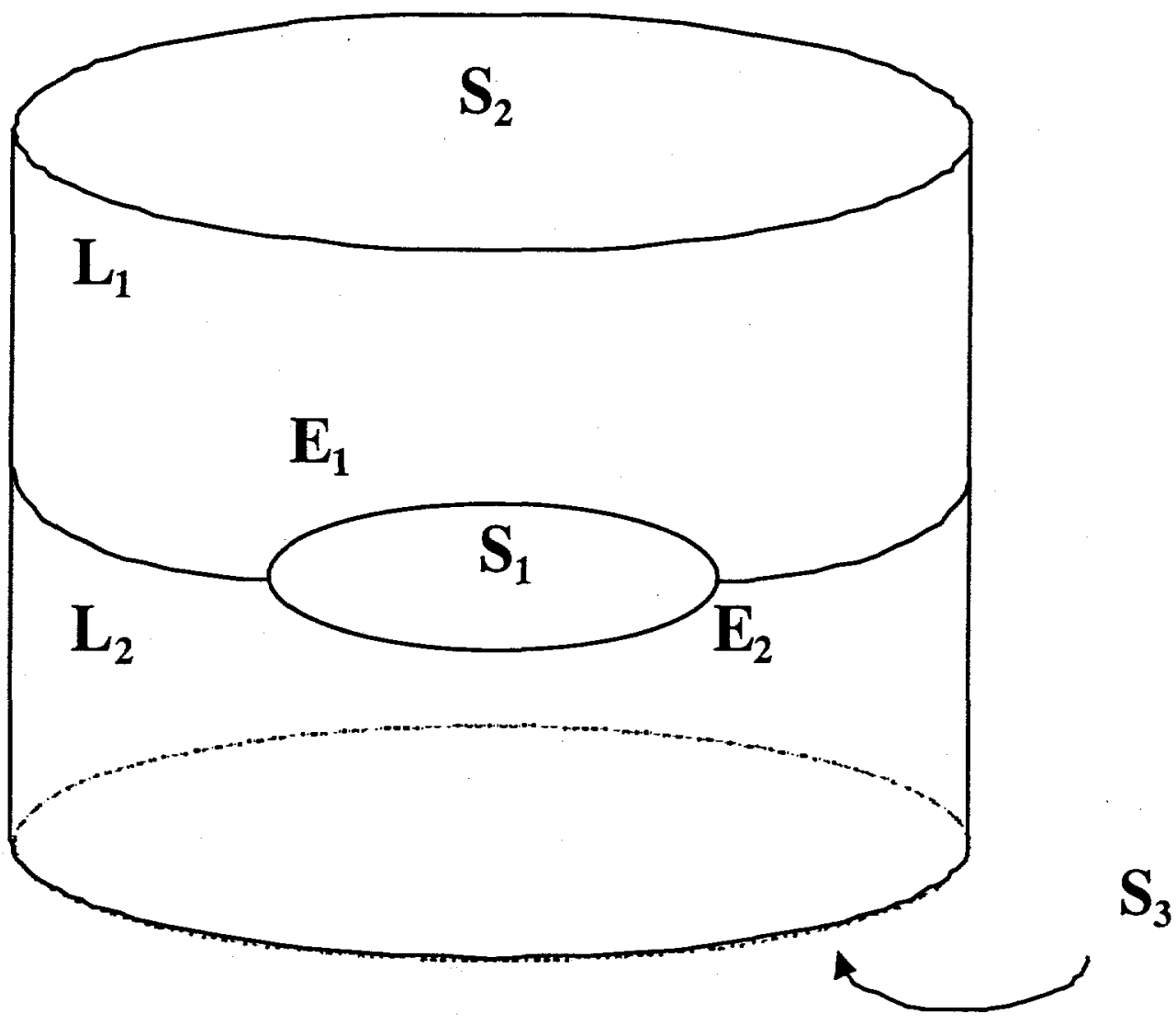

Figure 7: An example of Theorem $1 b$ and $1 c$ showing sweepability of a volume. When edges $E_{1}$ and $E_{2}$ are set to type End, the volume is not sweepable; the volume becomes sweepable if $E_{1}$ or $E_{2}$ is set to type corner. 
4

1

Figure 8: Vertex type adjustment for vertex 4 makes surface mappable (left); making surface mappable allows volume to be swept (right). 
Table 1: Pseudo code for traversing source/target surfaces.

- find a non-submapped surface $r$; insert $r$ in source/target list $s$; call prop_to_next_src_tgt $(r, s)$

- Procedure prop_to_next_src_tgt(Surface $r$, Src/Tgt List q):

- put $r$ in source/target 1 ist $q$

- for each edge $e$ in surface $r$ :

- find other surface sharing that edge, $s$

- if $s$ is already in $S, T$ or $L$, continue to next edge

- else if edge $e$ is an end or corner, call link_to_next_src_tgt $(r, e, q)$

- else if e is side or reversal type:

- if $e$ is a side type, choose $p=q$

- else if e is a reversal type, choose $p=$ opposite $(q)$

- call prop_to_next_src_tgt $(r, p)$

- Procedure link_to_next_src_tgt(Surface $r$, Edge e, src/Tgt List q):

- if $r$ is neither mapped nor submapped, or has no valid chains, return NOT_SWEEPABLE

- put $r$ in linking surface list $I$

- for each edge $f$ of surface $r$ :

- if edge $f$ has ij parameter different from that of $e$, continue at next edge

- get other surface $s$ sharing edge $f$

- if $\mathrm{S}$ is already in $\mathrm{S}, \mathrm{T}$ or $\mathrm{L}$, continue at next edge

- else if edge $f$ has type side, call link_to_next_src_tgt(s, $f, q$ )

- else:

- set type of next source/target surface $s$ by calling p=next_type (e, $f, q)$

- call prop_to_next_src_tgt $(s, p)$

Procedure next_type (Edge e, Edge f, Src/Tgt List p)

- If e and $f$ are different type (End-Corner or Corner-End), return p

- Else if e and $f$ are same type (End-End or Corner-Corner), return opposite (p), where opposite(SRC_LIST) = TGT_LIST, etc. 
Table 2: Pseudo code for building sweep dependencies.

- Collect all sweepable volumes and unmark them

- Collect all sweepable "end" volumes (volumes with source/target surface not shared by another sweepable volume)

- For each end volume $v$ :

- If $v$ is marked, continue to next volume

- Mark v

- Call prop_from_source(v, source_volume_list)

- Call prop_from_target(v, target_volume_list)

- Sweep_group = source_volume_list + target_volume_list

- If any unmarked sweeepable volumes, resolve cyclic sweep groups

Procedure prop_from_source (volume $\nabla$, volume list source_vol_list)

- Get source face list for $v$

- For each source face f:

- $a=$ adjacent volume from $v$ across $\mathrm{f}$

- If a is NULL, continue to next face

- Else if a is not assigned sweep scheme, continue

- Else if a is marked, continue

- Else

- Mark a

- Call prop_from_source (a, source_vol_list)

- Insert a in source_vol_list

- If $f$ is a source face for a:

- Call prop_from_target (a, source_vol_list)

Procedure prop_from_target (volume $v$, volume list target_vol_list)

- Get target Iist for $v$

- For each target face $f$ :

- $a=$ adjacent volume from $v$ across $f$

- If a is NULL, continue

- Else if a is not assigned sweep scheme, continue

- Else if a is marked, continue

- Else

- Mark a

- Call prop_from_source(a, target_vol_list)

- Insert a in target_vol_list

- Call prop_from_target(a, target_vol_list) 
Figure 9: Four volumes demonstrating sweep order dependencies. 

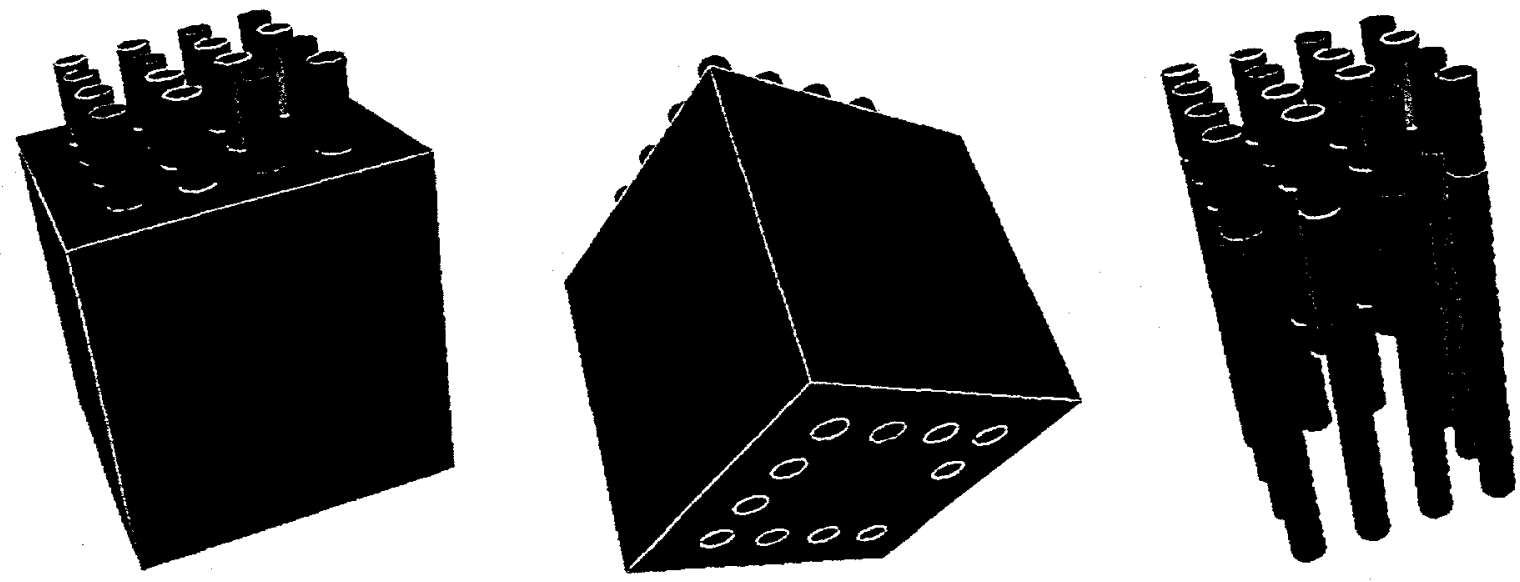

Figure 10: Collection of volumes whose schemes, source and target surfaces were detected automatically. Note inner core of rods which produces blind holes in block. 
Figure 11: Volume whose sweep scheme and source and target surfaces were determined automatically. 
Figure 12: Volume whose sweep scheme and source/target surfaces were determined automatically. 
Figure 13: Group of volumes whose sweep schemes and source and target surfaces were determined automatically. 